\title{
Impediments to implementation of real-time pathology prediction in Barrett's esophagus and colorectal polyps
}

Author

Institution

\author{
Douglas K. Rex
}

Division of Gastroenterology/Hepatology, Indiana University School of Medicine, Indianapolis, Indiana, United States submitted

18. February 2015 accepted after revision

20. February 2015

\section{Bibliography}

DOI http://dx.doi.org/

10.1055/s-0034-1391850

Published online: 24.6.2015

Endosc Int Open 2015; 03:

E186-E188

(c) Georg Thieme Verlag KG

Stuttgart · New York

E-ISSN 2196-9736

\section{Corresponding author}

\section{Douglas K. Rex}

Indiana University School of Medicine - Gastroenterology 550 N. University Blvd., Suite 4100

Indianapolis, Indiana 46202

United States

Fax: +1-317-944-5449

drex@iu.edu

\section{Abbreviations \\ $\nabla$ \\ ASGE American Society for Gastrointestinal Endoscopy \\ PIVI Preservation and Incorporation of Valuable endoscopic Innovation}

Tools like narrow band imaging (Olympus, Center Valley, Pennsylvania, United States), confocal laser microscopy (Mauna Kea Technologies, Paris, France), and chromoendoscopy (sometimes with magnification) have been used to successfully predict the pathology of diminutive colon polyps $[1,2]$ as well as the presence of dysplasia in Barrett's esophagus [3-8]. Despite numerous publications demonstrating that these and other tools can successfully predict pathology compared to white light endoscopy, the influence of such image enhancements in the routine clinical management of colorectal polyps and Barrett's esophagus has been limited. Colorectal polyps, even when diminutive (and with virtually no risk of malignancy), are resected and consistently sent to pathologists. This paradigm is costly and could be replaced by the more cost-effective practice of estimating polyp pathology in real time during colonoscopy, followed by resection of diminutive lesions without submission to the pathologist. This new paradigm for management of diminutive polyps, usually called resect and discard, was estimated to allow over one billion dollars per year in health care savings in the United States [9]. However, despite nearly 100 published trials showing that trained and interested colonoscopists can differentiate conventional adenomas from lesions in the serrated class [1], resect and discard has yet to enter clinical practice.

Similarly, endoscopists continue to manage Barrett's esophagus by examination of lesions in white light, and if no nodules are identified, they perform systematic biopsy at specified intervals, but otherwise without direction. In clinical prac- tice, these biopsies are frequently not obtained in the recommended number [10]. Further, in expert hands, image enhanced inspection and directed biopsy of Barrett's esophagus has been shown to improve the yield of dysplasia compared to white light biopsies that may be systematic in number and interval, but are not directed [3-8]. Clearly, it is sometimes difficult to implement new paradigms in endoscopic and other medical practices, even though evidence appears to support the paradigms and they pass the test of common sense.

In an attempt to salvage the clinical utility of floundering endoscopic technologies, including endoscopic imaging tools, The American Society for Gastrointestinal Endoscopy (ASGE) initiated a process it called Preservation and Incorporation of Valuable endoscopic Innovation (PIVI) [11,12]. The idea of the PIVIs was that experts would first delineate clinical problems where management could potentially be improved by application of a new endoscopic technology. Experts in the PIVI topic would define the most clinically relevant endpoints that should be addressed by investigators performing evaluations of the new technology. The PIVIs also established performance thresholds that should be achieved by the technology. The final step in the PIVI process is accompanied by an implied guarantee from the ASGE that if the performance thresholds are met, ASGE will endorse use of the new technology by appropriately trained and credentialed individuals as an acceptable alternative for the management of the originally defined clinical problem.

In this issue of Endoscopy International Open, Appannagari et al. remind us that incorporating new endoscopic management paradigms can be complex [13]. Approximately $20 \%$ of gastroenterologists responding to a survey indicated they would be unwilling to incorporate the management paradigm proposed in the ASGE Barrett's esophagus PIVI into their practice, because of concerns 
Table 1 Remaining measures to make real-time determination of pathology a viable clinical practice for diminutive colorectal polyp management and target biopsy in Barrett's esophagus. ${ }^{1}$

Professional society endorsement
Development of credentialing protocols
Development of validated training tools
Documentation of endoscopic decision making (image storage)
- Medical-legal coverage
- Documentation of adenoma detection rate ${ }^{2}$
Revision of institutional policies on requirements to submit tissue
to pathology ${ }^{2}$
Reimbursement or other financial incentives for endoscopic
determination of pathology
${ }^{1}$ Adapted from Rex, DK. Prediction of colorectal polyp pathologic lesions with image-
enhanced endoscopy: what will it take to make it matter? Gastrointest Endosc 2014;
80: 1088 - 1093 (reference number 14 ) with permission
${ }^{2}$ Applies only to colorectal polyps

about medical-legal risk and lack of financial incentives. Given that the survey was completed by either United States national meeting attendees (who may be more willing than average to try a new practice) or a tiny fraction (3.9\%) of the email recipients of the survey, we can reasonably conclude that $20 \%$ underestimates the resistance to incorporating the Barrett's esophagus management protocol proposed by the PIVI and based on image-enhanced endoscopy.

The Table 1 lists the primary remaining obstacles to implementation of the new imaging-based management paradigms proposed in the ASGE PIVIs for colorectal polyps and Barrett's esophagus $[11,12]$. The medical-legal concerns encountered in the study by Appannagari will vary between countries that have different medical-legal environments, between states and regions within the United States that have different laws and risk levels pertaining to medical-legal malpractice, and between practitioners who have different levels of concern about medical-legal matters. The solution to these medical-legal concerns is the availability of systems that provide long-term storage of high-definition images or videos that memorialize the evidence used for an endoscopist's real-time decisions regarding pathology [14]. This image storage is exactly analogous to storage of radiographic images and offers the consequent medical-legal risk and protection from risk afforded to radiologists by those images.

The issue of financial incentives is complex and sometimes linked to medical-legal risk. Many physicians reasonably question why they should undertake any practice that entails new medical-legal risk and has no financial rewards. In Japan, endoscopists now receive a payment (about $\$ 20$ USD) for documenting use of image-enhanced endoscopy. In the United States, there is now a physician billing code for the use of confocal laser microscopy, which in a strange twist is the real-time imaging technology with the highest costs for implementation, is the least practical, and takes the longest to learn. None of the other imaging tools (narrow band imaging, Flexible spectral Imaging Color Enhancement, iScan, autofluorescence, etc) have a billing code, and there is currently no opportunity for reimbursement. The trend in the United States has been progressive reductions in physician payments for endoscopy, and getting new payments from insurers for use of these technologies seems unlikely. The best scenarios for creation of financial incentives for image-based pathology predictions appear to be payment schemes like "reference" and "bundled" payments where endoscopists receive a fixed fee that includes payment for the physician's performance of the procedure, the facility fee, the sedation costs, and the pathology fees
[15]. In a bundled payment system, endoscopists are incentivized to use anesthesia specialists sparingly and to minimize pathology costs. Without reference or bundled payments, the financial and medical-legal disincentives to image-based pathology prediction are real and substantial and should not be underestimated. One need only look to the example of endoscopist directed propofol to appreciate the power of these factors. Endoscopist directed propofol was supported by extensive evidence [16], but created medical-legal risk for endoscopists while simultaneously it produced no financial incentives and even created disincentives.

There is a way forward. In my opinion, there are two key steps. The first key step is to acknowledge that when real-time imagebased management paradigms are endorsed by professional societies as effective and acceptable strategies for patient care, that does not mean they are established as the standard of care and must be used by every practitioner. Rather, endorsement of the technologies means that they are an accepted alternative to the current pathology-biopsy management paradigm. Clinicians will have the option of incorporating these new practices, and may choose to do so if the new paradigm matches their interest, training, expertise, credentialing, and financial incentives. Endorsement of the new paradigm by professional societies is also important as the other half of medical-legal protection, because it supports those practitioners who decide to use the new standard of care.

The second key step in moving forward is to transition the performance-based thresholds proposed in the ASGE PIVIs from technology-based thresholds to practitioner-based thresholds. This step is an important correction that responds to the reports of varying success in meeting the ASGE PIVI polyp thresholds and better fits with how quality in the technical performance of diagnostic tests or therapeutic procedures is judged in clinical practice. To elaborate, the PIVI threshold targets have been achieved for resect and discard in studies from academic centers $[2,17$, $18]$, but not in studies from private practice centers $[19,20]$. This creates concern that the paradigm cannot be successfully implemented in community practice and therefore should not be implemented at all. The reality is that there would be many disinterested faculty members within the academic centers that produced successful studies who did not participate in the trials, have not been trained in real-time polyp pathology interpretation, and should not be allowed to practice resect and discard. Conversely, in community practice there were some colonoscopists who successfully met the PIVI thresholds even though most of them did not [19]. Therefore, it makes sense to make resect and discard a valid practice for any individual endoscopist, regardless of the practice setting, who demonstrates that they can meet the PIVI thresholds. Moving to an individual practitioner-based competency model has the additional advantage of deemphasizing the competition between technologies. It is less important which technology a practitioner uses, as long as they can meet the PIVI thresholds with the technology available. In reality, this is how many endoscopic and surgical procedures are and should be judged. For example, regardless of which endoscopes are purchased by an endoscopy unit, each practitioner within that unit should have an adequate adenoma detection rate [21]. Similarly, if a practitioner wants to implement resect and discard, let them prove they are capable.

Everyone agrees that health care resources are spread increasingly thin. There are several adjustments to current colonoscopy practice that could reduce costs. For example, as adenoma detection rates increase with education and new technology, we could 
allow colonoscopists with high adenoma detection rates to use longer screening and surveillance intervals for low-risk colonoscopy findings. Similarly, as adenoma and polyp detection rates increase and newer colonoscopes permit detection of tiny lesions that may never harm patients, we could ask: "Are we going to continue to send every tiny polyp for an expensive pathologic assessment?" Current practice often defies common sense. Going forward, we need to demonstrate the will and courage to overcome impediments to best and most cost-effective patient care.

Competing interests: Dr. Rex receives research support and consulting fees from Olympus, United States.

\section{References}

1 Wanders LK, East JE, Uitentuis SE et al. Diagnostic performance of narrowed spectrum endoscopy, autofluorescence imaging, and confocal laser endomicroscopy for optical diagnosis of colonic polyps: a metaanalysis. Lancet Oncol 2013; 14: 1337-1347

2 McGill SK, Evangelou E, Ioannidis JP et al. Narrow band imaging to differentiate neoplastic and non-neoplastic colorectal polyps in real time: a meta-analysis of diagnostic operating characteristics. Gut 2013; 62: $1704-1713$

3 Sharma P, Weston AP, Topalovski M et al. Magnification chromoendoscopy for the detection of intestinal metaplasia and dysplasia in Barrett's oesophagus. Gut 2003; 52: 24-27

4 Sharma P, Meining AR, Coron E et al. Real-time increased detection of neoplastic tissue in Barrett's esophagus with probe-based confocal laser endomicroscopy: final results of an international multicenter, prospective, randomized, controlled trial. Gastrointest Endosc 2011; 74: $465-472$

5 Sharma P, Marcon N, Wani $S$ et al. Non-biopsy detection of intestinal metaplasia and dysplasia in Barrett's esophagus: a prospective multicenter study. Endoscopy 2006; 38: 1206-1212

6 Singh $R$, Karageorgiou H, Owen $V$ et al. Comparison of high-resolution magnification narrow-band imaging and white-light endoscopy in the prediction of histology in Barrett's oesophagus. Scand J Gastroenterol 2009; 44: 85-92

7 Wallace MB, Sharma P, Lightdale C et al. Preliminary accuracy and interobserver agreement for the detection of intraepithelial neoplasia in Barrett's esophagus with probe-based confocal laser endomicroscopy. Gastrointest Endosc 2010; 72: 19-24

8 Qumseya BJ, Wang H, Badie $N$ et al. Advanced imaging technologies increase detection of dysplasia and neoplasia in patients with Barrett's esophagus: a meta-analysis and systematic review. Clin Gastroenterol Hepatol 2013; 11: 1562 - 1570 [e1-e2]

9 Kessler WR, Imperiale TF, Klein RW et al. A quantitative assessment of the risks and cost savings of forgoing histologic examination of diminutive polyps. Endoscopy 2011; 43: 683-691

10 Abrams JA, Kapel RC, Lindberg GM et al. Adherence to biopsy guidelines for Barrett's esophagus surveillance in the community setting in the United States. Clin Gastroenterol Hepatol 2009; 7: 736 - 742 [quiz 10]

11 Rex DK, Kahi C, O'Brien $M$ et al. The American Society for Gastrointestinal Endoscopy PIVI (Preservation and Incorporation of Valuable Endoscopic Innovations) on real-time endoscopic assessment of the histology of diminutive colorectal polyps. Gastrointest Endosc 2011; 73: 419-422

12 Sharma P, Savides TJ, Canto MI et al. The American Society for Gastrointestinal Endoscopy PIVI (Preservation and Incorporation of Valuable Endoscopic Innovations) on imaging in Barrett's esophagus. Gastrointest Endosc 2012; 76: 252 - 254

13 Appannagari A, Soudagar A, Pietrzak $C$ et al. Are gastroenterologists willing to implement imaging surveillance for Barrett's esophagus? Results from a national survey Endoscopy International Open (in press)

14 Rex DK. Prediction of colorectal polyp pathologic lesions with imageenhanced endoscopy: What will it take to make it matter? Gastrointest Endosc 2014; 80: 1088-1093

15 Brill JV, Jain R, Margolis PS et al. A bundled payment framework for colonoscopy performed for colorectal cancer screening or surveillance. Gastroenterology 2014; 146: 849-853 [e9]

16 Rex DK, Deenadayalu VP, Eid E et al. Endoscopist-directed administration of propofol: a worldwide safety experience. Gastroenterology 2009; 137: 1229-1237 [quiz 518-519]

17 Rex DK. Narrow-band imaging without optical magnification for histologic analysis of colorectal polyps. Gastroenterology 2009; 136: $1174-$ 1181

18 Ignjatovic A, East JE, Suzuki N et al. Optical diagnosis of small colorectal polyps at routine colonoscopy (Detect InSpect ChAracterise Resect and Discard; DISCARD trial): a prospective cohort study. Lancet Oncol 2009; $10: 1171-1178$

19 Ladabaum U, Fioritto A, Mitani A et al. Real-time optical biopsy of colon polyps with narrow band imaging in community practice does not yet meet key thresholds for clinical decisions. Gastroenterology 2013; 144: $81-91$

20 Schachschal G, Mayr M, Treszl A et al. Endoscopic versus histological characterisation of polyps during screening colonoscopy. Gut 2014; 63: $458-465$

21 Rex DK, Schoenfeld PS, Cohen J et al. Quality indicators for colonoscopy. Gastrointest Endosc 2015; 81: 31-53 